\title{
CAMBIOS PERCIBIDOS POR PERSONAS CON DIVERSIDAD FUNCIONAL MOTORA ADQUIRIDA EN SUS VIDAS COTIDIANAS TRAS LA OBTENCIÓN DE PERROS DE SERVICIO.
}

\section{CHANGES PERCEIVED BY PEOPLE WITH ACQUIRED MOTOR FUNCTIONAL DIVERSITY IN THEIR DAILY LIVES AFTER OBTAINMENT SERVICE DOGS.}

\author{
Alejandra González $M^{1}$.Angélica Guerrero $A^{2}$., Damari Naranjo $G^{3}$., Francisca Rojas $V^{4}$., Carla Silva $F^{5}$., Carla Frías $O^{6}$.
}

\section{RESUMEN:}

El presente estudio surge del interés por analizar los cambios percibidos por personas con diversidad funcional motora adquirida en sus vidas cotidianas tras la adquisición de perros de servicio. La revisión de investigaciones internacionales sobre efectividad de la tenencia de estos animales, así como la revisión de las teorías del vínculo y de la biofilia proporcionan un primer acercamiento para comprender los posibles cambios. A partir de todo ello surge la pregunta: ¿Cómo perciben los cambios en sus vidas cotidianas las personas en estudio tras la obtención de perros de servicio otorgado por la corporación Bocalán Confiar? El estudio se visualiza desde el paradigma interpretativo y contempla la revisión teórica y fuentes directas a través de entrevistas semi-estructuradas, mediante las cuales se obtuvo una perspectiva personal acerca de la situación. Se analizaron los cambios desde nueve categorías: Actividades de la vida diaria, trabajo remunerado, tiempo libre, relaciones interpersonales, familia, accesibilidad, responsabilidad, seguridad y vínculo. Los hallazgos dejan en evidencia que la obtención del perro de servicio no sólo influye en independencia funcional, sino también en el ámbito emocional, social y familiar.

Palabras claves: Perros de servicio, diversidad funcional motora adquirida, vida cotidiana.

\section{ABSTRACT:}

This study arises from the interest in analyzing the changes perceived by people with motor functional diversity acquired in their daily lives following the acquisition of service dogs. The revision of international research on effectiveness of tenure of these animals, as well as the revision of the theories on bonding and biophilia provides

${ }^{1}$ Terapeuta Ocupacional, Universidad de Chile. Magister en Administración y Gestión en Salud, Universidad de los Andes. Profesor Asistente del Departamento de Terapia Ocupacional y Ciencia de la Ocupación, Universidad de Chile. Contacto: agonzalezmoreno@med.uchile.cl

${ }^{2}$ Terapeuta Ocupacional Hospital San Juan de Dios. Huérfanos 3255 RM Contacto: dreamy.angel@live.cl; Fono 225741900

3 Terapeuta Ocupacional Programa MAS Adultos Mayores Autovalente CORSABER El Bosque. O'Higgins 840 damari.naranjo@gmail.com; Fono 224209100

${ }^{4}$ Terapeuta Ocupacional Instituto de Rehabilitación Infantil. Avda. Bernardo O'Higgins 4620. fran.rv9@gmail.com; Fono 226772000

5 Terapeuta Ocupacional Programa MAS Adultos Mayores Autovalente COSAM San Joaquín. Santa Rosa 2606. c.silvafadda@gmail.com. Fono 228108409

${ }^{6}$ Licenciada en Trabajo Social, Pontificia Universidad Católica de Valparaíso. Doctora en Sociología, Universidad Autónoma de Barcelona, Máster en Investigación Aplicada (UAB). Máster de Políticas sociales y Desarrollo Local, UARCIS. Contacto: carlafrias@med.uchile.cl. 
insight to understand the possible changes. From all this, the question arises: How do the people under study perceive the changes in their daily lives after obtaining service dogs by Bocalán Confiar Corporation? The study is visualized from the interpretive paradigm and provides the theoretical review and direct sources through semistructured interviews, by means of which a personal perspective on the situation was obtained. Changes were analyzed from nine categories: activities of daily living, paid work, free time, relationships, family, accessibility, accountability, security and link. Findings evidence that the obtainment of the service dog does not only influences functional independence, but also in the emotional, social and family field.

Keywords: Service dogs, acquired motor functional diversity, daily life.

\section{INTRODUCCIÓN}

Este artículo pretende introducir al lector en la percepción que tienen las personas con movilidad reducida tras la incorporación de un perro de servicio en su vida cotidiana.

Las Personas con Diversidad Funcional Motora $(\mathrm{PcDFM})^{7}$ son aquellas que presentan “Alteraciones o deficiencias orgánicas del aparato motor o de su funcionamiento, presentando una clara desventaja (...) en relación con el promedio de la población, que se va a manifestar en posturas, desplazamientos, coordinaciones y manipulación”. (Chocomeli, Falcones y Sánchez, 2011 pp. 1). Estas personas pueden presentar limitaciones en el desempeño en su vida cotidiana, requiriendo asistencia en sus AVD, con la consiguiente repercusión personal, social y económica, tanto para ellas como para su familia (Henao y Pérez, 2010).

Se utiliza el concepto de PcDFM debido a la concepción que se tiene sobre los sujetos de estudio basándose en el modelo de diversidad funcional que surge desde el modelo social de la discapacidad o de vida independiente en España durante el año 2001, con el fin de suplir las carencias en el ámbito ético que presentaban los modelos anteriores, postulando tres grandes ideas: a) sustituir el término "discapacidad" por el de "diversidad funcional", b) aceptar las diferencias en torno a la funcionalidad como una forma más de diversidad inherente a la condición humana, c) reconocer la dignidad, otorgando el mismo valor a todas las vidas humanas, garantizando así los mismos derechos y oportunidades (Guzmán, Toboso y Romañach, 2010).

Entre las formas de asistencia convencional para PcDFM se encuentran el uso de silla de rueda, push adaptativo u órtesis, pero no son las únicas ayudas, ya que la asistencia basada en animales es otra alternativa. Animales como los perros son especialmente entrenados para asistir a personas con diversidad funcional en sus vidas cotidianas y/o como recurso terapéutico. Dichos perros se les conoce como perros de asistencia y se sub clasifican en: perro señal, guía, de alerta médica, de servicio para niños del espectro autista y para PcDFM.

Los animales, en especial los perros, han sido considerados como el "mejor amigo del hombre" dado que brindan a sus dueños/as compañía, cariño, seguridad y un sinfín de sentimientos que tienen

\footnotetext{
${ }^{7}$ Para facilitar la lectura y el buen uso del espacio en el texto se abrevia la frase Personas con Diversidad Funcional Motora con sus iniciales PcDFM
} 
Cambios percibidos por personas con diversidad funcional motora adquirida en sus vidas cotidianas tras la obtención de perros de servicio. distinto significado para cada persona. Sin embargo, no es hasta los años 70's que el psicólogo Boris Levinson se da cuenta que la compañía de estos animales impacta positivamente en el tratamiento de niños/as con trastornos del espectro autista, conduciendo a cambios emocionales positivos (Muñoz y Valero, 1999).

El aporte de los perros a la rehabilitación física llega de dos maneras fundamentales: primero, como motivadores durante las sesiones y segundo como ayudas técnicas para quienes poseen dificultades para realizar Actividades de la Vida Diaria (AVD), las que se entienden como "tareas de automantenimiento, movilidad, comunicación y manejo del hogar, que brindan independencia personal" (Pedretti, 1981 en Moruno, 2006 pp. 5).

En Chile aún son escasas las fundaciones y corporaciones que realizan entrenamiento a perros para que asistan a PcDFM. Una de ellas es la Corporación Bocalán Confiar, "una institución sin fines de lucro con personalidad jurídica otorgada por el Decreto Exento $N^{\circ} 3327$, cuyo objetivo es contribuir en el proceso de rehabilitación y en la inserción social de las personas con discapacidad a través de los animales" (Bocalán Confiar, 2003). La corporación dispone de un equipo permanente de personal capacitado como entrenadores de perros de asistencia y técnicos en terapia asistida, tanto en Chile como en el extranjero. Ellos realizan la selección de perros, los entrenamientos así como los seguimientos una vez entregados a las personas con diversidad funcional.

Esta corporación afirma que los perros de servicio deben transformarse en una ayuda técnica para quienes reciben la asistencia, la que se entiende como "elementos y/o implementos requeridos por una persona con discapacidad para prevenir la progresión de la misma, mejorar o recuperar su funcionalidad" (Art. 6 letra b Ley $\mathrm{N}^{\circ}$ 20.422). Por ende, dichos perros pasan a ser una ayuda fundamental para los/las PcDFM, asistiéndolos/as en la realización de sus AVD y facilitando su independencia.

Los perros de servicio, son animales entrenados para ayudar en actividades como alcance, movilización y vestimenta, facilitando la socialización e independencia de las personas con problemas motores (Oropesa, García, Puente, y Matute, 2009). Estos animales de servicio pueden llegar a ser una ayuda fundamental en el manejo dentro del hogar para las personas que lo requieren, mejorando la calidad de vida de los/as mismos/as (Henares, Calvo y Cruz, 2008).

En la actualidad han surgido nuevas perspectivas que van más allá de la consideración del perro de servicio como una ayuda fundamental en AVD e independencia (Haraway, 2003), plantea que no sólo proveen compañía o impactan en algún tipo de tratamiento, sino que además existe una coconstrucción entre el animal y la persona a cargo de él, generando una relación de interdependencia en la cual el animal ayuda a la PcDFM y éste a su vez cuida de él/ella.

Esta investigación recoge los aspectos mencionados y además realiza un análisis los cambios percibidos por cuatro PcDFM adquirida en sus vidas cotidianas tras la adquisición de sus perros de servicio.

En Chile, el 12,9\% de la población (1.048.347 personas) ${ }^{8}$ tiene diversidad funcional, de este porcentaje, el 31,3\% presenta diversidad funcional de tipo motora ENDISC (2004). Una de las principales causas de son los accidentes de tránsito, que ocupa el lugar número seis de las 25 causas específicas del indicador Años de Vida Ajustados por Discapacidad (AVISA) y las enfermedades

${ }^{8}$ Cifra obtenida desde última Encuesta Nacional de Discapacidad (ENDISC), año 2004. No se cuenta en Chile con datos actualizados 
cerebro vasculares ubicadas en el lugar once. Ambas condiciones conllevan una carga de muerte o diversidad funcional (Ministerio de Salud de Chile, 2008).

Es así, que surge el adiestramiento de animales para asistir a personas en sus vidas cotidianas y contrarrestar las necesidades que presentan las PcDFM derivadas de las limitaciones en el movimiento, tal como se menciona en estudios realizados por Pedro Oropesa y cols. (2009). En este contexto, los perros de servicio, que según la perspectiva dominante, y desde la que se posiciona Bocalán Confiar, son una ayuda técnica para el usuario/a permitiéndole conseguir funciones fundamentales como mejorar la movilidad (empujar silla de ruedas, abrir puertas, servir de apoyo, prender y apagar luces), alcanzar objetos y facilitar la socialización e independencia. Una característica relevante de estos animales es que no pueden distraerse bajo ninguna circunstancia y deben estar lo suficientemente capacitados para desenvolverse en cualquier contexto sin presentar ningún signo de agresividad. Las razas que se seleccionan para la asistencia son generalmente Labrador y Golden Retriever (Oropesa, García, Puente y Matute, 2009) debido a su docilidad (facilidad de adiestramiento), calma (excitabilidad baja), escasa agresividad, iniciativa, adaptabilidad en relación a las tareas en que se utilice y su alta capacidad para aprender comandos.

Para optar a un perro de servicio debe la persona ser adulta y con una disminución física, mínimo de destreza en manos y brazos que le impida llevar a cabo algunas o varias AVD (Instituto Canino Europeo, Canix., 2013), lo que se relaciona con una limitación en la independencia, como en el caso de PcDFM. Desplazarse, manipular objetos, bañarse, vestirse y asearse son actividades cotidianas que pueden verse afectadas. Cualquier factor que dificulte su realización o impida su desempeño implica algún grado de interdependencia con otros/as humanos, animales u objetos y no sólo una relación de "ayuda" o "asistencia".

Por los antecedentes expuestos es que surge el interés de indagar en los cambios percibidos por PcDFM adquirida tras la llegada de sus perros de servicio, reforzado por evidencias teóricas que afirman la existencia de cambios positivos tras la tenencia de estos animales en la realización de sus AVD. A pesar de dicha evidencia, no se ha considerado la subjetividad de cada persona, considerando que la vivencia de una persona a otra puede diferir enormemente. Por ello surge la siguiente interrogante: ¿Cómo perciben los cambios en sus vidas cotidianas las PcDFM encuestadas, tras la adquisición de perros de servicio?

Este artículo pretende contribuir en el ámbito social como aporte a una mirada diferente del término de discapacidad, utilizando el modelo de la diversidad que surge del modelo social de la discapacidad, el cual sustituye este mismo término por el de diversidad, otorgando el mismo valor a todas las vidas humanas en igualdad de condiciones y derechos en directa relación con aspectos legales pertinentes.

Además, el estudio contribuye con perspectivas subjetivas en torno a la temática, siendo un aporte para nuestro país, ya que se cuenta con una mirada global de la población por parte de instituciones que objetivan del tema, y no con las vivencias personales que son distintas dependiendo del contexto en el que se desarrolla la experiencia. 
Cambios percibidos por personas con diversidad funcional motora adquirida en sus vidas cotidianas tras la obtención de perros de servicio.

Desde la terapia ocupacional, es importante conocer la evidencia sobre los beneficios otorgados en las ocupaciones de personas que adquieren un perro de servicio, tanto en la facilitación de algunas actividades como en otras áreas de la vida cotidiana, tales como vida social y afectiva.

El objetivo general de la investigación es analizar los cambios que perciben cuatro PcDFM adquirida en sus vidas cotidianas tras la obtención de perros de servicio entregados por la Corporación Bocalán Confiar, entre los años 2003-2013 en la ciudad de Santiago.

Como objetivos específicos se establece:

- Describir los cambios que perciben en sus vidas cotidianas las personas en estudio luego de obtener los perros de servicio.

- Conocer los aspectos comunes y las diferencias sobre el cambio percibido en la vida cotidiana entre las personas estudiadas.

\section{MARCO CONCEPTUAL}

La vida cotidiana es entendida como "un conjunto multitudinario de hechos, de actos, objetos, relaciones y actividades (...) es decir, como acción, como mundo en movimiento" (Pampliega de Quiroga y Racedo, 2007 pp. 11). La misma autora menciona que los sujetos se relacionan entre sí y con la naturaleza de acuerdo a sus necesidades, en un tiempo y espacio determinado, configurándose así su cotidianidad, según refiere Pampliega de Quiroga y Racedo (2007) está constituida por

la familia en que nacimos, la familia que constituimos, la revista o el diario que leemos, la televisión, el cine, el teatro, el tipo de comida que cocinamos, el tipo de casa en la que habitamos, las alternativas de la moda, los medios de transporte, nuestra economía, el deporte, el arte que nos emociona, el tipo de relaciones sexuales que mantenemos. Vida cotidiana es la forma de desenvolvimiento que adquiere día tras día nuestra historia individual. (Pampliega de Quiroga y Racedo, 2007 pp. 11-12).

Es así como la vida cotidiana engloba a todas aquellas actividades que se realizan en el día a día, incluyendo las que involucran la interacción con otros/as. Pampliega de Quiroga menciona que hay tres esferas que conforman la vida cotidiana: la primera es el tiempo libre, entendido como la "Distensión de la carga laboral cotidiana" (Pampliega de Quiroga y Racedo, 2007 pp. 22-23) es decir, tiempo liberado de trabajo, de la monotonía y fatiga, o también definido como "actividad no obligatoria, que esta intrínsecamente motivada y en la cual se participa durante un tiempo discrecional o libre, es decir, un tiempo no comprometido con ocupaciones obligatorias tales como trabajo, cuidado propio o dormir" (Parham y Fazio, 1997, pp. 250 en Ávila, Martínez, Matilla, Máximo, Méndez, Talavera, Rivas y Viana, 2002).

La segunda esfera es el trabajo que según la autora es "una acción previamente planificada y social", no instintiva como en los animales, sino que diseñada y pensada con anterioridad, destinado además a 
satisfacer las necesidades vitales operando sobre la naturaleza y "transformando la realidad externa para alcanzar sus objetivos" (Pampliega de Quiroga y Racedo, 2007 pp. 23). Este concepto, ha sido también definido por otros autores como una "actividad con fines de producción o recompensa o aquella que sin ser asalariada contribuye a la subsistencia y producción. Generalmente se encuentra limitado por tiempo y espacio" (Hopkins y Smith, 1998 en Peñas, 2003).

Finalmente, la tercera esfera de análisis es la vida familiar, que es homologada con la vida privada, concebida como un lugar de refugio para cada persona, una tregua en la que se puede tener una pausa a la "hostilidad y exigencia del mundo laboral" (Pampliega de Quiroga y Racedo, 2007 pp. 21), "pero que implica responsabilidad, obligación, exigencia y trabajo doméstico" Pampliega de Quiroga y Racedo, 2007 pp. 23).

Entonces, se puede decir que estas tres esferas, sumado al conjunto multitudinario de actos, objetos, y actividades realizadas en el día a día dan forma a las denominadas ocupaciones, que según Kielhofner están referidas al "hacer del trabajo, juego o de las actividades de la vida cotidiana, dentro de un contexto temporal, físico o sociocultural que caracteriza gran parte de la vida humana" (Kielhofner, 2004 pp. 9). La profesión de terapia ocupacional usa el término de ocupación para capturar la naturaleza y el significado de "actividad diaria", encontrándose por ende bajo el término más amplio de vida cotidiana, al igual que las relaciones que se establecen en ella. Desde el Marco de Trabajo de la American Occupational Therapy Association (AOTA, 2010) estas ocupaciones se categorizan en áreas de la ocupación, que incluyen actividades de la vida diaria básicas, actividades de la vida diaria instrumentales, educación, trabajo, juego, ocio/tiempo libre, descanso/sueño y participación social.

Para efectos del estudio se consideran las áreas de trabajo, tiempo libre y actividades de la vida diaria por ser aquellas en las que la llegada del perro de servicio pudiese tener alguna repercusión según las referencias estudiadas. Las dos primeras ya han sido conceptualizadas con anterioridad desde Pampliega Quiroga y la AOTA, mientras que las AVD son aquellas que "están orientadas al cuidado del propio cuerpo" (Rogers y Holm, 1994 págs. 181-202 en Ávila, 2002) y "al apoyo en la casa y en la comunidad" (AOTA, 2010). Algunos ejemplos de las primeras son bañarse, alimentarse, tener movilidad funcional e higiene menor (Ávila y cols., 2002); mientras que de las segundas: el uso de tecnología, manejo de dinero y compras, cuidado de mascotas, movilidad en la comunidad, preparación de la comida y limpieza.

Es importante destacar el aspecto relacional que engloba el concepto de vida cotidiana planteado por Pampliega Quiroga, el cual va ligado directamente a otro de los aspectos que pudiese verse influenciado tras la adquisición de un perro de servicio: las relaciones interpersonales, entendidas como "contactos profundos o superficiales que existen entre las personas durante la realización de cualquier actividad" (Rodríguez, s.f. en Marcio y Ramos, 2012 pp. 29). Según Bisquerra (2003) son interacciones mutuas de dos o más sujetos, de relaciones socialmente establecidas reguladas por ámbitos legislativos e institucionales.

Desde el punto de vista de la relaciones y desde los lazos formados producto de éstas entre las personas es que se van configurando vínculos, comprendidas desde la teoría postulada por Pichón- 
Cambios percibidos por personas con diversidad funcional motora adquirida en sus vidas cotidianas tras la obtención de perros de servicio. Riviére (2000), en la que se consideran las relaciones sujeto-sujeto y sujeto-objeto. En este sentido, el animal es considerado por cada persona de la forma que sea significativa para ella; como una ayuda técnica (objeto) o como un integrante de la familia (sujeto). Esta teoría comprende al vínculo como algo relativamente fijo y que tiende a repetirse de manera automática, tanto en las relaciones interna como externas. Dentro del campo externo el sujeto tiende a relacionarse con otros sujetos u objetos que lo rodean, mientras que en el campo interno se establecen relaciones con las propias ideas, pensamientos, ensueños o fantasías. Para Pichón-Riviére el vínculo tiene una significación exclusiva para cada individuo, con componentes bidireccionales y de mutua afectación (Pichón-Riviére, 2000 en Hernán, s.f).

Para Katcher (1993) en Gutiérrez, Granados y Piar (2007) se necesitan cuatro principios básicos para poder formar el vínculo entre hombre-animal. Estos principios son: seguridad que guarda relación con la protección del animal hacia el ser humano, la tranquilidad que le otorga y la mejora en sus relaciones sociales, intimidad entendida como la comunicación entre humano-perro, las mutuas caricias y la habilidad que tienen los perros para comprender señales verbales y no verbales de las personas (Bentosela y Mustaca, 2006 en Gutiérrez, Granados, y Piar, 2007). Este vínculo que establecen las personas con los animales es respaldado desde la hipótesis de la biofilia entendida como "la afiliación innata emocional de los seres humanos hacia otras criaturas vivas" (Wilson 1984 en Krcmarova, 2009 pp. 2).

Estas relaciones otorgan una serie de beneficios en las áreas física, psicológica y social. En la primera se favorece la prevención de enfermedades y se facilita la rehabilitación (Wilson y Turner, 1998 en Gutiérrez, Granados, y Piar, 2007). En el área psicológica, ayuda a centrar la atención, mejora el sentido del humor, autoestima y las demostraciones emocionales con los demás (Beck 1997, Cusack, 1991, en Gutiérrez, Granados y Piar, 2007). Finalmente en el área social el animal genera un efecto catalizador que facilita al dueño/a el acercamiento a otras personas y mejoran la relación con ellas (Beck, 1997, Wilson y Turner, 1998, en Gutiérrez, Granados y Piar, 2007).

En relación a los beneficios y respecto a los perros de servicio, un estudio realizado en el contexto estadounidense por Rintala, y Seitz, (2008), presenta evidencia de que la utilización de perros de servicios otorga beneficios en la realización de AVD de los sujetos de estudio. Los/las participantes informaron tener un mejor desempeño en AVD tales como: cuidado personal, tareas de la casa, movilidad en el hogar y la comunidad. Sumado a esto los sujetos reconocen que los perros de servicio les ayudaron en su salud así como también a obtener más reconocimiento y aceptación social. Además, el perro de servicio puede otorgar autoconfianza, amistad y afecto (Canix, 2013).

En Chile debido a los beneficios demostrados se han formado instituciones para el adiestramiento de perros de asistencia, lo que ha llevado a que exista respaldo legal para regular el uso de perros guía o de servicio por parte de personas con diversidad funcional, promulgándose el 30 de Mayo del año 2005 la Ley 20.025 que modifica la Ley 19.284 (5 de enero del año 1994) que regulaba el acceso, la circulación y el cuidado de los perros de asistencia en compañía de PcDFM, estableciendo el acceso y derecho a permanencia de toda persona que utilice perro guía o de servicio en cualquier infraestructura o espacio de uso público, y derecho a circular en cualquier medio de transporte nacional ya sea público o privado, no estando sujeto a pago de dinero extra.

Para fines del estudio es pertinente definir tres conceptos; Independencia, interdependencia y autonomía: 
Independencia, se debe tener en cuenta que distintos autores han propuesto recientemente el término de interdependencia, que escapa en cierta medida de las tradicionales concepciones de apoyo y dependencia, mencionadas con anterioridad. Según Haraway, 2003 en Palli, 2006, los seres humanos no existen como entes individuales en el mundo, sino que están expuestos los unos a los otros en una relación de co-construcción, de mutua ayuda y mutua influencia.

Por otro lado el concepto de autonomía hace referencia a "la capacidad de tomar decisiones por sí sola/os y afrontar las consecuencias de ello de acuerdo a preferencias propias y a los requerimientos del entorno, aun cuando se necesita de la ayuda y apoyo de otras personas para lograrlo" (Comisión Económica para América Latina y el Caribe, CEPAL, 2012. pp. 7). Esta diferenciación de términos, se realiza para determinar el tipo de requerimientos que las personas del estudio poseen en cuanto a la capacidad de hacer actividades, la toma de decisiones y a las relaciones de mutua ayuda, con el fin de otorgar una mayor comprensión sobre la relación que establecen con sus perros de servicio.

Todas las personas de este estudio presentan lesión medular, que se define según la Organización Mundial de la Salud (OMS, 2013) como las injurias que recibe la médula espinal producto de traumatismos o enfermedades en la cual los síntomas dependen tanto de la gravedad del daño como de la localización de éste. De esta forma, la sintomatología puede conllevar la pérdida de sensibilidad y/o movimientos de algunas, o todas las extremidades y hasta de todo el cuerpo, además de repercutir en el desempeño en su vida cotidiana. Es así como los perros de servicio pueden facilitar tareas que requieran de movimientos activos de segmentos afectados, tales como alcances, abrir/cerrar puertas y/o vestirse, influyendo positivamente en la independencia funcional de la persona.

\section{METODO}

\section{Criterios de Inclusión}

La muestra es intencionada, y seleccionada según los siguientes criterios de inclusión:

- Persona mayor de 18 años.

- Poseer diversidad funcional motora adquirida.

- Recibir perro de servicio otorgado por la Corporación Bocalán Confiar.

Dentro de los criterios de exclusión se encuentran:

- Expresión oral alterada.

- Poseer daño cognitivo.

Dentro de los aspectos éticos es importante mencionar que se respeta a cada uno de los sujetos en estudio y que se entrega a todos la información sobre el proceso y objetivos de la investigación. Para formar parte del estudio las personan debían conocer y firmar, si así lo deseaban, un consentimiento informado, mediante el cual queda de manera explícita su libre participación, resguardo de la 

confidencialidad y anonimato. Para mantener el anonimato de los/as entrevistados/as se nominan los perros con la letra $\mathrm{P}$ y el número del entrevistado/a que corresponda: P1, P2, P3, P4.

Esta investigación es de carácter cualitativo y se posiciona desde el paradigma interpretativo, el cual entiende la naturaleza de la realidad, como una interpretación o una comprensión de los sujetos en estudio desde múltiples realidades construidas (G. Briones, 1996). Por esto es que la recolección de datos se realiza mediante entrevistas semi-estructuradas.

El instrumento utilizado para la recolección de los datos obtenidos corresponde a una creación del equipo de trabajo, para dar cuenta de las Actividades de la Vida Diaria, Trabajo Remunerado, Tiempo Libre, Relaciones Interpersonales, Familia, Accesibilidad a servicio y a uso de espacios públicos, Responsabilidad, Seguridad y Vínculo con el perro de servicio, tomando los elementos contemplados en el marco teórico para su elaboración y que dan cuenta de las percepciones y significados de cada uno de los sujetos entrevistados. Por tanto, las aproximaciones de cada uno/a de ellos/ellas serán diferentes, porque su vivencia así lo es.

La comprensión dinámica y diversa de la realidad conlleva a que el comportamiento de los sujetos no está determinado por características generales, existiendo una comprensión particular de los fenómenos explorados.

Al tratarse de un estudio narrativo se recolecta información de un aspecto de la historia de vida de los/as participantes para describirla y analizarla, considerando tanto a la persona como a su entorno (Salgado, 2007).

Al ser un diseño exploratorio descriptivo indaga sobre los cambios percibidos en la vida cotidiana de los participantes, una vez que han recibido su perro de servicio. Asimismo, describe la visión de un fenómeno determinado, el cual es caracterizado a través de relatos de los propios protagonistas, presentando un panorama de esta situación de manera individual, en donde estos describen los cambios mencionados con anterioridad.

Luego de una etapa de selección de los participantes, se configuran los sujetos de estudio, de la siguiente manera:

Tabla 1

Perfil de Personas Incluidas en el Estudio

\begin{tabular}{|c|c|c|l|l|}
\hline \multicolumn{5}{|c|}{ Perfil de los entrevistados/as } \\
\hline E & Edad & DFM & \multicolumn{1}{|c|}{ Trabajo } & Vive con \\
\hline 1 & 34 & Tetraplejia & $\begin{array}{l}\text { Locutora } \\
\text { radial } \\
\text { Docente }\end{array}$ & Pareja \\
\hline 2 & 46 & Tetraplejia & $\begin{array}{l}\text { Asistente } \\
\text { social }\end{array}$ & $\begin{array}{l}\text { Esposa } \\
\text { Dos hijos }\end{array}$ \\
\hline 3 & 65 & Paraplejia & Diseñador & Solo \\
\hline 4 & 36 & Paraplejia & $\begin{array}{l}\text { Sin trabajo } \\
\text { remunerado }\end{array}$ & $\begin{array}{l}\text { Madre } \\
\text { Primo } \\
\text { Esposa de } \\
\text { primo }\end{array}$ \\
\hline
\end{tabular}




\section{RESULTADOS}

La información obtenida a través de las entrevistas en relación a los cambios percibidos por los/as usuarios/as en sus vidas cotidianas, ha sido dividida y analizada de manera inductiva en nueve categorías para simplificar la comprensión de los resultados. Ocho de ellas se extraen directamente desde el marco teórico y la restante emerge de las propias entrevistas (responsabilidad). Es importante mencionar que estas categorías están íntimamente ligadas las unas con las otras, pese a ser analizadas por separado, dada la indivisibilidad del ser humano, por lo que sus ocupaciones, emociones, ideologías e intereses implicados no pueden ser aislados en la vida cotidiana de cada uno. A continuación se expone el análisis de los resultados de cada una de las categorías analizadas:

\section{Actividades de la Vida Diaria}

Área fundamental para la cual son entrenados los perros por parte de la corporación y en la que se espera que tenga mayor asistencia en la vida cotidiana de las PcDFM tal como lo menciona Rintala y Seitz (2008).

Los/as cuatro usuarios/as entrevistados/as identifican que el perro de servicio ha tenido influencia en la ejecución de sus AVD, simplificando la realización de actividades, a través de la facilitación de uno de los pasos que se requiere para lograr realizar la tarea: el alcanzar y recoger objetos.

Cuando se me caen cosas muy por allá que no alcanzo, P3 me las recoge y me las trae. P3 me abre las puertas, le tengo un sistema con un cordelito (...) le digo puerta y abre, me apaga y me enciende la tele de allá, principalmente esas cosas (E3).

Esto se traduce en una disminución del tiempo en realización de la tarea, un ahorro de energía para ellos/as y también para las personas que solían asistirlos/as en la realización de algunas actividades previo a la adquisición de los perro de servicio. "Al final las cosas las hago igual pero me cuesta más y me demoro más. P3 me ayuda y ahí si me demoro menos" (E3).

Se ha constatado que los perros de servicio asisten a los/as usuarios/as principalmente en acciones como recoger y alcanzar objetos (Bocalán Confiar, 2013). Esto permite comprender que dos de los/as entrevistados/as refieren sentirse más independientes en acciones que facilitan actividades, como ver televisión y llamar por teléfono ( 2 y 4 respectivamente). "Simplemente más independiente (...) antes tenía que estar llamando: "mamá pásame las llaves, mamá pásame esto". (...) Ahora con P4 aquí dentro de mi pieza, me muevo y me ayuda. Eso cambió mi independencia y no estar pidiendo ayuda a cada rato" (E4).

Por otro lado, los/as entrevistados/as 1 y 3 describen que sus perros, además los/as ayudan en tareas como abrir/cerrar puertas y cajones facilitando la movilidad intrahogar. De manera particular E1 describe que en caso de ser necesario su perro de servicio lo/a ayuda a desvestirse. "La otra vez me 
Cambios percibidos por personas con diversidad funcional motora adquirida en sus vidas cotidianas tras la obtención de perros de servicio. ayudó a sacar la parca, cuando lo necesito lo hace". En el caso de E3, su perro facilita el encendido/apagado del televisor. En relación a este último punto, la facilitación de la tarea mencionada puede contribuir a un enriquecimiento de otras ocupaciones como en este caso, de tiempo libre (Ávila y cols., 2002).

\section{Trabajo Remunerado}

Debido a que los/as entrevistados/as ya presentaban un trabajo remunerado antes de la adquisición del perro de servicio esta área no se ha visto mayormente influida en la obtención y ejecución, pero sí en la compañía y en el clima positivo que provocan. En el caso de E2 el perro de servicio realiza acompañamiento en diversas organizaciones por razones laborales, mientras que a E1 lo/a acompaña en el desarrollo de clases universitarias/locución radial, además refiere que su perro cambia el clima laboral en el que se desenvuelve y propicia un ambiente más grato. "Entonces Pl esté donde esté cambia el ambiente. Los lugares donde voy siempre, en la radio o en la facultad, genera impactos súper positivos" (E1). De modo particular E4 refiere que antes de la llegada de su perro de servicio, no se encontraba realizando una actividad remunerada por razones personales que no guardan relación con la adquisición de su perro de servicio. Por otro lado, a E2 el animal le facilita el alcance de objetos para la realización de la ocupación. "Me ayudó a alcanzar alguna cosa que no podía llegar porque la silla no cabía en los escritorios, así que yo le pedía que me los trajera o salíamos a terreno a visitar a organizaciones". (E2).

A partir de esto, se constata que los/as entrevistados/as no reportan cambios importantes en el área laboral, teniendo en cuenta que los perros de servicios pueden facilitar algunas actividades que requieran de alcances durante el trabajo. Ello justifica que hipotéticamente la incorporación del perro de servicio sí tendría un mayor impacto en esta área. Sin embargo, en estos casos analizados no se evidencia.

\section{Tiempo Libre}

En ésta área los/as entrevistados/as 1 y 2 describen que sus perros de servicio han creado nuevos espacios de tiempo libre junto a sus familias, relacionado principalmente a paseos en áreas verdes.

Tienes que hacerte el tiempo para salir con P2, independiente que se fuese a divertir a la plaza uno igual aprovechaba de pasarla bien" "Antes no lo hubiera hecho porque me hubiera dedicado a puro trabajar, pero ahora ya sé que no es sólo estar enfocado en el trabajo, uno también debe dejar sus espacios en cosas que a uno le gustan (E2).

A su vez los/as entrevistados/as 1 y 3 refieren que acoplan a sus perros en sus rutinas de tiempo libre, como por ejemplo ir a un restaurant, tomar un café o en caminatas. "Nosotros salimos porque queremos salir con P1, queremos ir a un parque, queremos salir a tomarnos un café con ella y queremos que salga, que camine, que haga ejercicio, tirarle la pelota, esos espacios los ha generado ella" (E1). Es así como esta categoría se liga de manera directa con las categorías de familia y acceso a servicio/uso de espacios públicos, mencionadas más adelante.

\section{Relaciones Interpersonales}

Las relaciones interpersonales es un área donde los/as entrevistados/as mencionan haber experimentado cambios importantes. Esto porque los perros de servicio actúan como facilitadores 
sociales. Tres de los/as entrevistados/as concuerdan que tras la llegada de sus perros de servicio se han abierto nuevos espacios sociales, principalmente en espacios públicos y uso de servicios, donde han conocido más personas y han podido establecer temas de conversación con los demás.

Yo creo que para el resto de la gente verme con un perro era novedad y sí, te abre nuevos espacios de conversación, nuevos espacios de conocer gente porque el sólo hecho de andar con P2 te atrae mucha gente a preguntarte, en qué te ayuda, cómo se puede conseguir (E2).

Esto puede ocurrir porque los perros seleccionados para hacer trabajo de servicio a PcDFM presentan características que llaman la atención de los/as demás e inspiran la confianza para acercarse a ellos. Incluso E3 refiere tener nuevas amistades desde la llegada de su perro de servicio, además de considerarlo como un catalizador social.

Aqui ha llegado gente nueva, generalmente jóvenes, mujeres principalmente y se acercan a P3, le hacen cariño y después se acercan a mí. Yo he hecho tres o cuatro amistades nuevas aqui por P3, porque si no tuviera a P3 sería una persona más en silla de ruedas, pero se me acercan, se acercan a P3 y de ahi se acercan a mí. Es una de las cosas que más ha cambiado en mi estado de antes de P3 y después de P3. Es un catalizador social total, además que son perros tan amorosos (E3).

Por otra parte, al igual que en el ámbito laboral, los perros de servicio generan cambios a nivel del clima social. E1 refiere que las personas cambian su actitud cuando lo/a ven con su perro de servicio.

Tú te subes al metro y la cara de la gente cambia, es como oh el perrito, en lugares públicos en general, lo mismo, entonces la gente te trata de otra manera, no te ven como la persona en silla de ruedas. Los problemas sociales que he tenido con P1 es el criterio de algunos taxistas y no me he hecho ningún problema y los he denunciado (E1).

Resulta interesante comprender estas relaciones interpersonales desde la teoría del vínculo de Pichón Riviére presentada en el marco teórico. En este sentido, el perro actúa como facilitador de relaciones sujeto - sujeto y ayuda a configurar nuevos vínculos y estrechar los ya existentes, como por ejemplo en la familia.

\section{Familia}

Se extrae de las entrevistas que la llegada del perro de servicio influye tanto al usuario/a como a todo el círculo familiar al que se integra. En el caso de E1, esto se evidencia en que ha disminuido el estrés y la preocupación de su pareja por cuidar de ella.

Mi pareja estaba trabajando en la ciudad empresarial y estaba muy preocupada que saliera más tarde y yo estuviera acá. Entonces venía en el metro con el Whatsapp, “¿estás bien?, pasa esto, pasa tal cosa, ¿necesitas algo?”; vivía en constante tensión. Se forma por tanto este nivel de estrés donde piensa: algo va a pasar si ella 
Cambios percibidos por personas con diversidad funcional motora adquirida en sus vidas cotidianas tras la obtención de perros de servicio. no llega. Hoy no, le digo si mi hermano ya se fue, pero estoy bien. Le da confianza también (E1).

Por su parte E4 refiere sentir mayor tranquilidad cuando su perro de servicio se queda en casa con su madre, porque sabe que ella no estará sola: “a mi mamá también le hace harta falta. Hay veces que yo no estoy en la casa en todo el día y P4 se queda con mi mamá, yo sé que está acompañada, yo sé que está bien" (E4).

En relación al resto de los/as entrevistados/as, E3 reconoce que su perro de servicio se ha vinculado fuertemente con sus hijos/as; "mis hijos la adoran, dicen que el día que yo no esté, si P3 sigue viva, ellos se van a quedar con ella porque la quieren mucho" (E3). Por el contrario E2 describe que el mayor cambio en su vida tras la llegada de su perro de servicio ha sido en esta área, ya que antes no compartía mayor tiempo con su familia, sino más bien se dedicaba plenamente al área laboral; en la actualidad los lazos familiares se han estrechado, compartiendo espacios de recreación juntos:

Rompió un esquema familiar que teníamos. Llegó un integrante nuevo a la familia, los niños estaban fascinados en ese tiempo, mis hijos eran chicos, y sabían que era un perro de trabajo, pero también un amigo más en donde depositar su cariño. (...) Evidentemente en su momento yo tenía que trabajar y puse todo mi esfuerzo en trabajar pero olvidé otras cosas, como salir con mis hijos al parque, olvidé un poco hacer más vida familiar. (...) Está bien trabajar, trabajar y estudiar, pero también hay que dejar espacios para el grupo familiar. (...) Hice cosas que antes de tenerlo a él nunca hice, como ir a una plaza con mi hijo a no sé, a comerte un helado, cuando lo sacábamos a pasear a él aprovechaba con mis dos hijos, le tirábamos juguetes, corría, en el fondo nos divertíamos como familia (E2).

Desde lo anterior se puede decir que todo el grupo familiar forma un vínculo con el perro, lo que puede derivar de la tendencia innata de unirse a un ser vivo, como lo menciona la hipótesis de la biofilia. Además, al ser el animal significativo para un integrante de la familia, también se inicia el proceso de vinculación con los demás integrantes por la visualización de la influencia positiva en el ser querido.

\section{Accesibilidad a Servicio y a Uso de Espacios Públicos}

En relación a la accesibilidad a servicios como son la locomoción colectiva, ingreso a restaurantes, centros comerciales y supermercados, los/as entrevistados/as identifican en común situaciones problemáticas que apuntan a la negación del acceso a éstos junto a sus perros. "Mis problemas que he tenido son cuando salgo con P3, los taxis no paran porque no tienen idea" (E3). En este mismo sentido, E2 refiere:

Es el desconocimiento de la gente, sobre todo en supermercados o restaurantes en donde ponían obstáculos para poder acceder con P2. (...) Igual a la gente le costaba aceptar que el perro entrara, pero esto no es un tema de capricho (...) sino que esto está respaldado por la ley (E2).

Tras la adquisición del perro los/as entrevistados/as pueden haber vivenciado experiencias conflictivas en los servicios mencionados a las que antes no estaban expuestos/as, teniendo que explicar en ocasiones la normativa legal para acceder a ellos, donde antes no se cuestionaba su ingreso.

Los/as entrevistados/as refieren estar informados/as sobre esta normativa (Ley 20.025). E4 menciona que la corporación ha contribuido de manera importante en el cumplimiento de la ley y 
fundamentalmente; ha entregado las herramientas necesarias para que los/as usuarios/as defiendan sus derechos:

Si tengo que pelear por P4 yo peleo, en un supermercado o en algún lugar que no permitan el acceso con ella. (...) En eso la fundación te entrega hartas herramientas; que no le tengas miedo a nadie. Además que es una ley, son tus derechos (E4).

Con respecto al uso de espacios públicos y la interacción con los transeúntes, se desprende de las entrevistas que existe un desconocimiento de la gente sobre la función de los perros de servicio, concibiéndolos en ocasiones como mascotas e interrumpiendo la labor que realizan, al acercarse por ejemplo a acariciarlos. Esto en oportunidades dificulta el desempeño de los/as mismos/as entrevistados/as en la movilidad dentro de la comunidad. En otras ocasiones la presencia del perro facilita el tránsito en silla de ruedas en los espacios públicos. "P3 por ejemplo, cómo anda siempre al lado mío va como abriendo camino, me facilita mucho el tránsito de mi silla de ruedas"(E3). Sin embargo, puede potenciar las relaciones interpersonales en el contexto fuera del hogar mencionadas anteriormente.

Se puede percibir en ésta área en general una falta de empatía por parte de la población, obstaculizando muchas veces el desplazamiento de las personas, situación que podría atribuirse al desconocimiento de las dificultades de movilidad presentes en las PcDFM. Esto se potencia aún más con la restricción al tránsito libre junto a su perro de servicio, por lo que resulta esencial una difusión masiva sobre el tema y generar más espacios con diseños que permitan el acceso a todos.

\section{Responsabilidad}

Todos/as los/as entrevistados/as indican que tras la llegada del perro han asumido una nueva responsabilidad, ya que es un ser vivo que está a su cargo y requiere de cuidados para sobrevivir. Sin embargo, estas actividades no son vistas como carga, debido al componente afectivo y de interdependencia que se da en la relación, haciendo de la experiencia algo positivo. Referente a esto, E2 menciona: "en el momento de adquirirlo uno asume una responsabilidad tremenda, porque independiente que yo lo necesite, P2 también me necesita como para yo cuidar de sus enfermedades, de bañarlo, de darle comida".

La tenencia del perro de servicio restablece la organización de las rutinas de los/as usuarios/as, tras la incorporación de nuevas actividades en relación a los cuidados que se deben tener con el animal. E3 y E4 refieren ser ellos/as mismo/as los que realizan las actividades de cuidados básicos de sus perros:

$P 3$ vive aquí conmigo adentro, tengo un patio atrás donde hace sus necesidades. Yo baño a P3 (...) con P3 viene una preocupación más, tener que verla, que quiere salir, darle la comida, recogerle su caquita, tener limpio el patio, una preocupación más que tengo, y eso me ayuda (E3).

Por otro lado E1 y E2 refieren que sus familiares cooperan en estas tareas tomándolo como una responsabilidad común. 
Cambios percibidos por personas con diversidad funcional motora adquirida en sus vidas cotidianas tras la obtención de perros de servicio.

Un concepto que puede desprenderse de esta categoría es la adquisición de un nuevo rol, el de cuidador, entendido rol como comportamientos socialmente esperados y determinados por la cultura (AOTA, 2010), al cual los/as participantes les atribuyen un significado positivo.

\section{Seguridad}

Desde las entrevistas se extrae que la seguridad está relacionada con dos aspectos centrales: el acercamiento de objetos en momentos en que se encuentran sin una persona que los pueda asistir (E1 y E4) y la compañía dentro y fuera del hogar (E2 y E3).

E1 refiere que una de las áreas que tiene mayor importancia, en relación a los cambios en su vida cotidiana es la seguridad, ya que el estar tranquilo/a estando solo/a le ha posibilitado aumentar su independencia.

La verdad es que dependía mucho de que hubieran personas conmigo para poder estar en la casa tranquila (...). Me costó mucho sentirme segura en mi propia casa (...) yo tenía que dejar la puerta abierta porque si me caía, pasaba cualquier cosa no podía abrir la puerta, si se me caía el celular estaba frita, no tenía quien me lo entregara porque estaba sola. Entonces yo creo que una de las principales cosas que me pasaba antes de tener a Pl era esta sensación de inseguridad (...) O sea ahora estoy sola, en vez de estar 45 minutos estoy 4 horas. Y eso es un cambio grande. Me da esa sensación de tranquilidad (E1)

Desde el punto de vista de la compañía, E3 refiere:

me da seguridad, ando acompañado ese es el tema. (...) Me ha tocado ir solo a algunos lugares y me siento desprotegido, me siento limitado. Cuando ando con alguien, con mi hija por ejemplo, me siento más acompañado, y con P3 también, de la misma manera (E3).

Esto lo reafirma E4, pero en el contexto intra domiciliario.

\section{Vínculo Usuario/a - Perro de Servicio}

Esta categoría es transversal a todas las demás por lo mencionado en los ámbitos de familia, responsabilidad significada positivamente y en general, la interdependencia que se desarrolla en todos los ámbitos de la vida de los/as entrevistados/as. Estos/as expresan haber formado un vínculo significativo con los animales, incluso desde el inicio del proceso de acoplamiento. E4 refiere "desde un principio yo supe que P4 era mía, desde el primer día en que yo la conocí (...) es como una conexión bien extraña, es como que nos miramos así y como que nos conocíamos". En este sentido, este primer acercamiento a los perros es crucial para establecer cuál es el animal con el que se tiene mayor conexión y afinidad.

Es importante destacar el cambio a nivel afectivo que ha habido en los/as entrevistados/as tras la adquisición de los perros, siendo muy significativa la presencia de estos a lo largo de todo el transcurso del día, compartiendo espacios íntimos de la vida cotidiana y llenando espacios vacíos. E1 lo plasma de la siguiente manera:

Te cambia la vida a nivel emocional tremendo, ya no nos imaginamos la vida sin P1 y lo hemos conversado en más de una oportunidad no nos imaginamos entrar a la casa y que no esté P1. Se sentiría vacio. El lazo emocional es muy fuerte (E4). 
E4 expresa que su perro de servicio es un importante y significativo apoyo emocional:

"P4 anímicamente es una gran ayuda. Todos esos momentos de amargura o de soledad los paso con P4; si tengo que llorar, lloro con P4. Yo creo que me da mucho apoyo. Aunque a mí no me habla, yo sé que ella está ahi'. (E4)

También es importante la compañía que brinda el perro de servicio para E3, expresando que Después que murió mi mamá estuve solo muchos meses, pero cuando llegó P3 yo sabía que había alguien que me estaba acompañando, P3 siempre trata de estar al lado mío, donde esté yo; echadita, siempre mirándome, pendiente. (...) En ese sentido me cambió la vida, la compañia (E3).

Finalmente cada uno de los/as entrevistados/as identificó un área de mayor importancia, relacionada con el cambio que han percibido en sus vidas. Dentro de estas categorías destaca el vínculo, la seguridad y la familia, que son áreas que están ligadas a lo emocional y relacional, quedando en evidencia que los cambios en las vidas cotidianas de PcDFM van más allá de la asistencia funcional.

\section{DISCUSIÓN}

En base a las entrevistas y a los análisis de las respuestas entregadas se obtienen los siguientes hallazgos: son múltiples los cambios en la vida cotidiana que los/as entrevistados/as perciben, luego de haber adquirido sus perros de servicio. Esto queda en evidencia en aquellas actividades (recoger celulares, abrir/cerrar cajones y puertas) en que previamente requerían asistencia de terceros, las cuales han sido suplidas por el perro, otorgándoles a las personas entrevistadas mayor independencia ${ }^{9}$.

Si bien estos animales son entrenados para funciones especiales como alcanzar/recoger objetos, abrir/cerrar puertas y cajones, contribuyendo en la independencia al facilitar el desarrollo de una actividad, también acompañan en momentos de soledad, transformándose en apoyos emocionales. Ligado a lo anterior, se evidencia un aumento en la sensación de seguridad al estar permanentemente con sus dueños. Es así, como la PcDFM depende de su perro de servicio y a la vez éste depende de él/ella para cubrir sus necesidades básicas de alimentación y cuidado, estableciéndose una relación de interdependencia. Esto, porque con los refuerzos positivos entregados al animal, más los cuidados y cariño permiten que el perro continúe realizando las funciones para las cuales fueron entrenados.

A su vez, el perro forma parte de la vida cotidiana de la persona, acoplándose en cada una de sus ocupaciones. Por esta mutua ayuda se configura el vínculo que influye en todas las categorías analizadas de la vida cotidiana de las PcDFM, siendo el eje transversal en esta relación de coconstrucción. De acuerdo a esto último, impresiona que los/as entrevistados/as no conciban al perro

\footnotetext{
${ }^{9}$ Se habla de independencia comprendiendo que todos/as los/as participantes son autónomos al poder decidir sobre acciones previo a la llegada del perro de servicio.
} 
Cambios percibidos por personas con diversidad funcional motora adquirida en sus vidas cotidianas tras la obtención de perros de servicio. de servicio como una ayuda técnica o un objeto, sino como un compañero, confidente y/o un integrante más de la familia. Esto genera cuestionamientos referente a cómo la subjetividad de cada persona influye en la forma de significar al perro dentro del vínculo. Frente a esta dualidad, surge la interrogante: ¿Es el perro de servicio un objeto o un sujeto?

Dentro de los análisis de resultados se destacan entre los/as entrevistados/as ciertos aspectos comunes y diferentes que responden a los cambios percibidos buscados en el estudio. Ejemplo de los primeros es el vínculo configurado entre los usuarios/as con el perro y entre la familia con el perro y el aumento de la independencia en las AVD, mediante la facilitación de pasos dentro de una actividad. Además, para todos/as los/as usuarios/as la llegada del perro ha significado la adquisición de un nuevo rol de cuidador y de nuevas responsabilidades para otorgar los cuidados necesarios al animal, influyendo directamente en la modificación de la rutina diaria, especialmente en los espacios de tiempo libre, momentos en los cuales pueden realizar nuevas actividades junto al perro, por ejemplo sacarlo a pasear.

Finalmente, otro aspecto común encontrado en el estudio, es el hecho de que el perro ha actuado como un catalizador social para establecer nuevas relaciones interpersonales y abrir nuevos temas de conversación en distintos contextos, con el consiguiente beneficio que ello trae para las personas.

En relación a los aspectos diferentes, como lo es el trabajo remunerado, el cambio está directamente relacionado al tipo de trabajo realizado, influyendo más en los que requieren actividades de alcance de objetos. Esta última acción repercute también en el aspecto de la seguridad para los/as usuarios/as, expresándose de diversas maneras: a unos/as les otorga más independencia, por ejemplo en momentos de emergencia mientras que a otros/as, compañía en situaciones en las que realizan actividades sin ayuda de terceros.

Pese a los beneficios que pueden ser descritos por los/as entrevistados/as en sus vidas cotidianas, todos/as han mencionado dificultades en torno al acceso a servicios y uso de espacios públicos, a pesar del respaldo legal que existe en torno a esto, lo que puede ser atribuido al escaso conocimiento sobre la verdadera importancia e influencia que tiene un perro de servicio para las PcDFM y la falta conciencia social al no permitir el libre desplazamiento de cualquier persona en los espacios públicos. Muchos siguen viendo a estos animales como "mascotas" y no como un animal en trabajo de servicio, quedando en evidencia la falta de cumplimiento de los decretos que establece la Ley 20.025. Si bien, existe información acerca de la temática, ésta se encuentra concentrada en ciertos servicios especializados en temas de diversidad funcional haciéndose necesaria la difusión masiva sobre el tema al resto de la población.

En relación a esto, se puede apreciar cómo día a día las personas del estudio sufren de discriminación por esta falta de información, teniendo dificultades para el acceso a recintos públicos y/o privados, al no permitirles el ingreso con animales, sin comprender, muchas veces, que son perros de trabajo que facilitan la vida a la persona que acompañan, siendo un derecho transitar de manera libre en su compañía.

Desde la terapia ocupacional, se puede contribuir a esta labor de sensibilización, difundiendo los derechos de las PcDFM en la práctica cotidiana de la profesión. Además, se puede aportar información sobre la influencia positiva en la vida cotidiana de los/as usuarios/as en aspectos ocupacionales, fomentando este tema de interés en los actuales y futuros terapeutas ocupacionales, con el fin de crear una cadena de conciencia sobre estos beneficios que fomente el trabajo con este tipo de asistencia. Sumado a esto, el perro de servicio sirve como apoyo en la labor de terapia 
ocupacional, favoreciendo a una mejor calidad de vida mediante la facilitación de las tareas de la vida cotidiana, por ende puede ser visto como una herramienta terapéutica para la profesión.

Por lo mismo se propone como desafío a futuros profesionales del área contribuir con este tipo de estudios para PcDF no necesariamente motora y ampliar el campo de conocimiento sobre los beneficios ocupacionales que entregan los perros de asistencia en general y en las sub-clasificaciones. Se propone así, mirar desde otro prisma los actos concretos aportados por la teoría, es decir, como el simple alcance de objetos pueden contribuir a reforzar la autoeficacia, vínculos y ahorro de tiempo para tener más de éste para la ejecución de diversas actividades significativas para ellos/as mismos/as.

\section{REFERENCIAS BIBLIOGRAFICAS}

Ávila, A., Martínez, R., Matilla, R., Máximo, M., Méndez, B., Talavera, M., Rivas, N. y Viana, I. (2010). Marco de Trabajo para la Práctica de Terapia Ocupacional: Dominio y Proceso. 2 da ed. Recuperado de http://www.terapia-ocupacional.com/aota2010esp.pdf

Briones, G. (1996). Epistemología de las ciencias sociales. Bogotá, Colombia. ICFES.

Comisión Económica para América Latina y el Caribe, CEPAL. (2012). Autonomía e independencia: el cuidado de las personas con discapacidad. Cap.5. Recuperado el 10 de Octubre de 2014 desde http://www.cepal.org/publicaciones/xml/5/48455/PanoramaSocial2012DocICap5.pdf

Chocomeli, M., Falcones, A. y Sánchez, M. (2011). Digitalización de contenidos docentes para el Máster de Profesorado de Secundaria, en la asignatura Atención al Alumnado con Necesidades Educativas Especiales y uso de recursos Google en la docencia. Proyecto de Innovación Docente en la UMH. Recuperado el 20 de Octubre de 2013 desde http://ocw.umh.es/ciencias-de-lasalud/Atencion-al-alumnado-con-necesidades-educativas-especiales-459/materiales-deaprendizaje/unidad-7-la-diversidad-funcional-motorica/unidad-7.pdf

Corporación Bocalán Confiar. (2013). Quienes somos. Recuperado de http://www.bocalanconfiar.cl/quienes.html

Gutiérrez, G., Granados, D. y Piar, N. (2007). Interacciones humano animal: Características e implicaciones para el bienestar humano. Bogotá Colombia. Recuperado el 20 de Junio de 2014 desde http://www.revistas.unal.edu.co/index.php/psicología/article/view/1013/1469

Guzmán, F., Toboso, M. y Romañach, J. (2010). Fundamentos éticos para la promoción de la autonomía: Hacia una ética de la interdependencia. Alternativas, 17, 45 - 61. Recuperado el 11 de octubre de $2013 \quad$ desde http://riberdis.cedd.net/bitstream/handle/11181/3711/fundamentos_eticos_promocion_autonomia.pdf ?sequence $=1 \&$ rd $=0031949728317094$ 
Henao, C., Pérez, J. (2010). Lesiones medulares y discapacidad: revisión bibliográfica. Recuperado el 20 de agosto de 2013 desde http://www.scielo.org.co/pdf/aqui/v10n2/v10n2a06.pdf

Henares, P., Calvo, A. y Cruz, M. (2008). Un arma terapéutica alternativa: perros entrenados para asistencia o terapias. SEMERGEN Medicina de Familia 34 (04). Recuperado de http://zl.elsevier.es/es/revista/semergen-medicina-general-familia-40/articulo/un-arma-terapeuticaalternativa-perros-13119398

Hernán, H. (s.f). Sobre la teoría del vínculo en Enrique Pichón-Rivière. Una sistematización del texto Teoría del vínculo de Pichón-Rivière. Recuperado el 20 de junio del 2014 desde http://www.funlam.edu.co/uploads/facultadpsicologia/578481.pdf

Instituto Canino Europeo Canix. (2013). Perros de Servicio. Recuperado de http://canix.es/es/perros/servicio.htm

Kielhofner, G. (2004). Terapia Ocupacional Modelo de Ocupación Humana: Teoría y aplicación. 3ra. ed. Buenos Aires: Médica Panamericana.

Krcmarova, J. (2009). E.O. Wilson's concept of biophilia and the environmental movement in the USA. Klaudyán 6(1). pp.1-2. Recuperado el 20 de junio de 2014 desde http://www.klaudyan.cz/dwnl/200901/01_Krcmarova_pdf.pdf

Marcio, R. Ramos, c. (2012). Relaciones interpersonales en la empresa. Panamá. [Recuperado el 5 de Septiembre del 2014 desde: http://www.youblisher.com/p/270981-Relaciones-interpersonales-enla-empresa/]

Ministerio de Planificación de Chile. (2005). Ley 20.025: Modifica la ley 19.284, con el objeto de regular el uso de perros guías, de señal o de servicio por parte de personas con discapacidad. $\begin{array}{lllllll}\text { Recuperado el } & 20 & \text { de } & \text { junio } & \text { de } & & \text { desde }\end{array}$ http://www.leychile.cl/Navegar?idNorma=239523\&idVersion=2005-06-29

Ministerio de Salud de Chile. (2008). Estudio AUGE. Recuperado el 26 de octubre del 2013 desde http://epi.minsal.cl/epi/html/invest/cargaenf2008/Presentacion.pdf

Moruno, P. (2006). Actividades de la Vida Diaria. Barcelona: Masson

Muñoz Lasa, S. y Valero Alcaide, R. (1999) Los animales como ayudas técnicas. Rehabilitación 33(6). Recuperado el 10 de octubre de 2013 desde http://zl.elsevier.es/es/revista/rehabilitacin120/articulo/los-animales-como-ayudas-tecnicas-

13004975?referer=buscadorhttp://zl.elsevier.es/es/revista/rehabilitacin-120/articulo/los-animalescomo-ayudas-tecnicas-13004975

Organización Mundial de la Salud. (2013). Centro de Prensa: Lesiones Medulares. Recuperado de http://www.who.int/mediacentre/factsheets/fs384/es/

Oropesa, P., García, I., Puente, V. y Matute, Y. (2009). Terapia asistida con animales como fuente de recurso en el tratamiento rehabilitador. MEDISAN; 13(6) Recuperado el 10 de Octubre de 2013 desde http://bvs.sld.cu/revistas/san/vol13_6_09/san15609.html 
Palli, C. (2006) Diferencias que importan: Haraway y sus amores perros. Recuperado de http://www.raco.cat/index.php/Athenea/article/viewFile/53147/61121

Pampliega de Quiroga, A. y Racedo, J. (2007). Crítica de la vida cotidiana, (7ma. ed). Buenos Aires, Argentina: Ediciones Cinco.

Peñas, O. (2003). Aproximación crítica a la terapia ocupacional en la era de la información. Revista Chilena de Terapia Ocupacional. 3 (1). Recuperado el 05 de Septiembre de 2014 desde http://www.revistaterapiaocupacional.uchile.cl/index.php/RTO/article/view/152/133

Rintala, D., Matamoros, R. y Seitz, L. (2008). Effects of assistance dogs on persons with mobility or hearing impairments: A pilot study. Journal of Rehabilitation Research y Development 45(4). $\begin{array}{lllllll}\text { Recuperado el } & 12 & \text { de } & \text { Julio } & \text { del } & 2013 & \text { desde }\end{array}$ http://www.rehab.research.va.gov/jour/08/45/4/pdf/rintala.pdf

Salgado, A. (2007). Investigación cualitativa: Diseños, evaluación del rigor metodológico y métodos. Recuperado de file:///C:/Users/acer/Downloads/Dialnet-InvestigacionCualitativa-2766815.pdf

Biblioteca del Congreso Nacional de Chile (2010). Ley 20422 Recuperado de http://www.leychile.cl/Navegar?idNorma=1010903\&buscar=20422 